\title{
AVALIAÇÃO DA PRESCRIÇÃO DIETÉTICA DE CRIANÇAS HOSPITALIZADAS
}

\author{
ASSESSMENT OF DIETETIC ORDER IN HOSPITALIZE INFANTS
}

Maria Olímpia R V Almada'1, Lidiane B F Vilela¹, Cristina M M Resende', Jacqueline P Monteiro²

${ }^{1}$ Mestranda. ${ }^{2}$ Docente. Departamento de Pediatria. Faculdade de Medicina Ribeirão Preto - USP

Correspondência: Maria Olímpia Ribeiro do Vale Almada. Rua São José, 443 apto72 CEP: 14010-160 Ribeirão Preto-SP. Tel.: (16) 37973316 - (16) 91164427. e-mail: madovale@hotmail.com / madovale@yahoo.com.br

Almada MORV, Vilela LBF, Resende CMM, Monteiro JPM. Avaliação da prescrição dietética de crianças hospitalizadas. Medicina (Ribeirão Preto) 2007;40 (2): 255-9.

RESUMO: Modelo do estudo: Estudo de prevalência. Objetivo: A subnutrição é freqüentemente observada em crianças hospitalizadas e está associada a um pior prognóstico. A correta abordagem nutricional, incluindo a prescrição dietética, melhora a evolução clínica. O objetivo desse estudo foi avaliar a adequação da prescrição dietética em crianças hospitalizadas. Métodos: Vinte e uma crianças foram acompanhadas de Julho a Setembro de 2004. Foi realizado a avaliação das prescrições dietéticas de crianças recebendo nutrição enteral durante três períodos da internação. Resultados: Houve inadequação do peso durante a hospitalização (dia $1=$ 76.4 土 18.2\%; dia 2 = $76.1 \pm 18.6 \%$; dia 3 = $76.7 \pm 19.6 \%$ ), sem alteração durante o período do estudo. A prescrição energética foi inadequada, porém as de proteínas e micronutrientes apresentaram-se dentro da recomendação. Conclusão: A condição clínica pode afetar de maneira negativa a prescrição nutricional. A equipe multidisciplinar deve se empenhar ao máximo para atingir as necessidades energéticas das crianças hospitalizadas.

Descritores: Desnutrição. Criança Hospitalizada. Prescrição Dietética.

\section{1- INTRODUÇÃO}

A subnutrição é frequentemente encontrada em pacientes hospitalizados e está associada a um pior prognóstico ${ }^{1}$. A subnutrição infantil continua a ser um dos problemas mais importantes de saúde pública do mundo atual, devido à sua magnitude e aos conseqüentes prejuízos para o crescimento, desenvolvimento e sobrevida da criança ${ }^{2,3}$. Estudo demonstra que em países desenvolvidos, a subnutrição atinge de 15 a $20 \%$ dos pacientes hospitalizados. Em países como o México, têm-se encontrado até $80 \%$ de subnutrição entre as crianças hospitalizadas, observando-se, adicionalmente, um agravamento da condição nutricional durante o período de hospitalização ${ }^{4}$.

No Nordeste do Brasil, as formas graves de subnutrição energético-protéica chegam a atingir $24 \%$ das crianças menores de 5 anos de idade, hospitalizadas $^{5,6}$. A letalidade hospitalar em menores portadores de formas graves de subnutrição energético-protéica chega a ser quase dez vezes mais elevada quando comparada com crianças eutróficas ${ }^{5,7,8}$.

A importância da oferta de um suporte nutricional adequado é verificada ao se observar a prevalência de pacientes subnutridos hospitalizados. Considerando-se ainda que doentes subnutridos apresentam tempo mais prolongado de hospitalização e maior índice de morbidade e mortalidade que os eutróficos, independente da doença de base, a relevância do problema torna-se mais evidente. Por outro lado, as taxas de mortalidade são menores em pacientes que recebem a adequada terapia nutricional ${ }^{9,10}$. 
O desenvolvimento da dieta enteral, como um tipo de terapia nutricional eficaz, seguro e econômico, torna possível minimizar, talvez até evitar o problema da subnutrição energético-protéica em pacientes hospitalizados ${ }^{9,11}$.

Tendo em vista tais problemas, o objetivo deste trabalho foi avaliar a prescrição dietética da nutrição enteral de crianças hospitalizadas nas enfermarias do Hospital das Clínicas da Faculdade de Medicina de Ribeirão Preto da Universidade de São Paulo (HCFMRP/USP) no intuito de contribuir para um melhor planejamento de ações destinadas a minimizar a subnutrição hospitalar, proporcionando melhor recuperação e menor tempo de hospitalização.

Considerando os altos índices de subnutrição em crianças hospitalizadas encontrados na literatura é possível encontrar uma inadequação de peso (peso/ idade $<80 \%$ ) e uma inadequação da ingestão de energia $(<70 \%)$ nas crianças avaliadas.

\section{2- CASUÍSTICA E MÉTOdos}

Foi realizado um levantamento das prescrições dietéticas de crianças internadas no Hospital das Clinicas da Faculdade de Medicina de Ribeirão Preto da Universidade de São Paulo (HCFMRP/USP). O estudo foi aprovado pelo Comitê de Ética em Pesquisa da Instituição e os pacientes e/ou seus responsáveis diretos concordaram, por escrito, em participar do mesmo. Foram analisadas apenas as que recebiam dietas via enteral entre os meses de Julho e Setembro de 2004 ou seja $50 \%$ das crianças internadas nessa época. As prescrições dietéticas foram avaliadas em três momentos da internação: inicial, mediano e final; cada um desses momentos equivale, respectivamente, à primeira prescrição a partir da data de inclusão no estudo, à prescrição no meio do período de acompanhamento de estudo e à última prescrição do período do estudo. Posteriormente analisadas através de um software nutricional. As prescrições foram avaliadas quanto à energia e aos seguintes nutrientes: proteína, cálcio, ferro, fósforo, magnésio, potássio, sódio e zinco. A partir da quantidade prescrita de cada nutriente foi calculada a adequação de acordo com a Recommended Dietary Allowances (RDA/89) e Dietary References Intakes (DRI/03) ${ }^{12,13}$, para cada faixa etária. Foram considerados inadequados os valores $=80 \%$ de adequação $^{14}$.

Além da prescrição, foi analisada a adequação do peso/idade (NCHS) ${ }^{15}$ para cada momento da aná- lise. Subnutrição foi definida quando a criança apresentava valores $\leq 90 \%$ da adequação de peso ${ }^{16}$. Esses dados foram digitados em um banco de dados e analisados por programa EPINFO versão 6.0. Inicialmente, as variáveis contínuas serão testadas para verificar se tem ou não distribuição normal. As variáveis contínuas com distribuição normal serão expressas em termos de média \pm desvio padrão. O teste "t" nãopareado de Student será utilizado na comparação entre dois grupos, com eventual correção de Bonferroni. As variáveis contínuas sem distribuição normal serão expressas em mediana e valores mínimos e máximos; neste caso o teste de Wilcoxon Mann-Whitney será empregado na comparação entre dois grupos.

As proporções serão comparadas por meio do teste do $\chi^{2}$ (qui-quadrado) ou pelo teste exato de Fisher ${ }^{16}$.

Os resultados serão considerados significativos quando a probabilidade de rejeição da hipótese de nulidade for menor que $5 \%(\mathrm{p}<0,05)$.

\section{3- RESULTADOS}

Foram estudadas 21 crianças na faixa etária entre 1 a 169 meses, sendo que 13 eram do sexo masculino e 8 do sexo feminino. A faixa etária predominante foi abaixo dos doze meses de idade (61.9\%).

Os diagnósticos encontrados foram: Cardiopatias Congênitas (14,29\%); Leucemia Mielóide Aguda (4,76\%); Subnutrição grau III (4,76\%); Carcinoma Linfoepitelial de Nasofaringe (4,76\%); Insuficiência Renal Crônica (4,76\%); Intestino Curto (14,29\%); Doença de Hirshprung (4,76\%); Hipoplasia Adrenal (4,76\%); Anoxia Neonatal (9,52\%); Choque Séptico $(4,76 \%)$; Bronqueolite Obliterante (9,52\%); Diabetes Mellitus (4,76\%); Síndrome Ascítica (4,76\%); Dismotilidade Intestinal $(4,76 \%)$ e Doença do Refluxo Gastresofágico $(4,76 \%)$.

A Tabela I apresenta a adequação de peso, prescrição de energia, proteína e micronutrientes. Observa-se inadequação de peso das crianças hospitalizadas, e esta não teve alteração durante o período do estudo. A oferta energética esteve aquém das necessidades nos três momentos, a protéica apresentou-se acima da recomendação e a de micronutrientes esteve adequada, destacando-se o cálcio e o zinco que apresentaram-se acima do recomendado. A porcentagem de adequação da oferta de energia, proteína, sódio, potássio, cálcio, fósforo, magnésio, ferro e zinco não teve diferença significante durante o estudo. 
Tabela I: Adequação do peso e da prescrição de energia e de nutrientes das crianças hospitalizadas

\begin{tabular}{lllll}
\hline Variáveis* & Dia 1 & Dia 2 & Dia 3 & Valor P \\
\hline Idade (meses) & $8,3(1,03-169)$ & $9,09(1,09-169)$ & $10(2,00-169)$ & 0,84 \\
Peso (em quilos) & $6,8(2,8-49,4)$ & $7(2,5-50,2)$ & $7,5(2,7-50,2)$ & 0,95 \\
\% Adeq. de peso & $76,4 \pm 18,2$ & $76,1 \pm 18,6$ & $76,7 \pm 19,6$ & 0,99 \\
\% Adeq. de energia & $61,1 \pm 40,9$ & $60,8 \pm 43,3$ & $66,84 \pm 42,9$ & 0,87 \\
\% Adeq. de proteína & $120,4 \pm 96,2$ & $113,0 \pm 96,5$ & $122,4 \pm 92,9$ & 0,94 \\
\% Adeq. de sódio & $105,2(2-1397,8)$ & $99,7(2-2737)$ & $112,6(2-5403,6)$ & 0,77 \\
\% Adeq. potássio & $92,2 \pm 49,5$ & $90,5 \pm 53,0$ & $99,1 \pm 53,3$ & 0,85 \\
\% Adeq. cálcio & $149,5 \pm 81,0$ & $145,4 \pm 88,7$ & $161,7 \pm 84,5$ & 0,80 \\
\% Adeq. fósforo & $103,2(0,8-614,4)$ & $112,6(0,8-614,4)$ & $130,2(0,8-350)$ & 0,66 \\
\% Adeq. magnésio & $102,7 \pm 84,4$ & $99,4 \pm 86,9$ & $99,0 \pm 72,8$ & 0,98 \\
\% Adeq. ferro & $149,4(14,9-2544)$ & $103,4(15,3-2544)$ & $99,3(5,4-3181)$ & 0,96 \\
\% Adeq. zinco & $187,7(69-640)$ & $187,7(24,6-2082)$ & $214(66,6-402)$ & 0,82 \\
\hline
\end{tabular}

\section{4- DISCUSSÃO}

Neste estudo pode-se observar que além da inadequação do peso no primeiro momento da avaliação, as crianças não obtiveram ganho de peso ao longo do período. Isso se deve tanto a oferta inadequada de energia, quanto à doença de base que pode requerer uma necessidade energética maior. E, apesar da oferta protéica apresentar-se acima do recomendado, a falta de energia suficiente para o melhor metabolismo protéico, torna-a inadequada ao crescimento dessas crianças.

O peso em todos os momentos do estudo encontrou-se abaixo de $90 \%$ da adequação, o que pode implicar em um pior prognóstico da criança. Em um estudo conduzido em Bangladesh com 437 crianças gravemente subnutridas, de 12 a 60 meses, verificouse recuperação nutricional em regime de internação, hospital-dia e domiciliar. Os autores encontraram que o tempo para atingir a recuperação nutricional foi significativamente menor no grupo de pacientes interna$\operatorname{dos}^{17}$.

Em um trabalho realizado por Oliveira et al. ${ }^{8}$, com 125 crianças subnutridas pode-se observar que a melhora do estado nutricional esteve intimamente ligada à adequada ingestão energética durante a internação e que as crianças que ingeriram mais do que uma vez a recomendação de energia por quilo de peso para a idade foram as que tiveram melhor evolução nutricional. No nosso estudo, a oferta energética insuficiente nos três momentos da internação pode ser um fator etiológico para a inadequação do ganho do peso.

O excesso de proteína e cálcio, avaliados neste trabalho, pode ser justificado pela dieta a base de leite. Manary et al. ${ }^{18}$, avaliando 53 crianças marasmáticas durante infecção aguda, observaram que uma alta oferta protéica e isoenergética, melhora a síntese de proteínas corporais e de resposta de fase aguda, quando comparada a uma oferta normoprotéica ${ }^{18}$. Contudo observamos que uma oferta aumentada de proteína em conjunto com uma dieta hipocalórica, não melhora o estado nutricional de crianças hospitalizadas, visto que essa proteína em excesso será desviada para produção de energia.

Apesar da oferta de ferro nesta dieta estar adequada, o excesso de cálcio pode interferir na disponibilidade desse micronutriente, conforme comprovado por Hallbert et al. ${ }^{19,20}$, que observaram uma diminui- 
ção em $60 \%$ da absorção de ferro quando se ingeria leite e derivados durante as principais refeições ${ }^{19,20}$.

No entanto, Thorsdottir et al. ${ }^{21}$, em 2003 demonstraram que essa associação só é negativa se a oferta de cálcio for muito elevada. Porém GrinderPederson et al. ${ }^{22}$, afirmam que não há diminuição da absorção de ferro não-heme, quando se oferece uma dieta fortificada em cálcio.

O excesso da ingestão oral de zinco em nível de toxidade é raro. Entretanto uma suplementação contínua de zinco bem acima da DRI pode interferir na absorção do cobre. Em pacientes com deficiência renal ou em hemodiálise, a toxicidade de zinco é mais comum e é caracterizada por anemia, febre e distúrbios do sistema nervoso central.

Os inúmeros diagnósticos encontrados podem ser um fator limitante deste estudo, pois foram avaliadas somente vinte e uma crianças internadas na enfermaria que recebiam apenas dieta enteral. As necessidades podem ser diferentes devido à diversidade de patologias encontradas. A oferta hídrica pode também explicar a inadequação energética, pois quando restrita pode se tornar uma barreira a uma oferta adequada de energia ${ }^{15,23}$. Alcançar as necessidades elevadas de calorias e proteínas, com rigorosa restrição hídrica, torna-se um verdadeiro desafio para o suporte nutricional e metabólico adequado dessas crianças. Assim o nutricionista deve maximizar os esforços no sentido da modulação das dietas e do uso de suplementos para aumentar a densidade energética da dieta sem aumentar seu volume, oferecendo assim energia suficiente para o "catch-up" do crescimento. Os dois produtos utilizados com maior freqüência para aumentar a densidade energética das fórmulas infantis são os triglicérides de cadeia longa e média e os polímeros de glicose.

O tempo de internação foi outro fator que pode ter interferido na avaliação do peso e da oferta energética, devido à diversidade deste período para cada criança. Os dias da coleta de dados também foram um fator limitante, pois poderiam ter coincidido com momentos de piora da condição clínica, além de não podermos afirmar que toda a prescrição dietética tenha sido ingerida.

Outra limitação do presente estudo é a inclusão de pequena amostragem com diagnósticos diversos, não sendo suficiente para classificá-los conforme a patologia.

Conforme previsto os dados obtidos permitem concluir que a prevalência do déficit de peso para a idade em crianças hospitalizadas foi bastante elevada. A equipe multidisciplinar deve se empenhar para minimizar a inadequação energética das crianças hospitalizadas a fim de colaborar para a redução do tempo de hospitalização e para melhora do prognóstico da criança.

Almada MORV, Vilela LBF, Resende CMM, Monteiro JPM. Assessment of dietetic order in hospitalize infants. Medicina (Ribeirão Preto) 2007;40 (2): 255-9.

ABSTRACT: Background:Malnutrition is frequently found in hospitalized children and is associated with poor outcome. The adequate nutritional approach improves clinical evaluation. The aim of this study was to evaluate the adequacy of dietetic enteral order in hospitalized children. Methods: Twenty one children were followed from july to september 2004. The dietetic enteral orders were registered during three periods of hospitalization. Results: The children body weight was inadequate and didn't changed during the hospitalization (day $1=76.4 \pm 18.2 \%$; day $2=76.1$ $\pm 18.6 \%$; day $3=76.7 \pm 19.6 \%$ ). Energy dietetic order was inadequate but protein and micronutrients were according to the recommendation. Conclusion: Clinical condition may negativelly affect the nutritional order. The multidisciplinary team should give all the efforts to meet the appropriate dietetic energy order of hospitalized children.

Keywords: Malnutrition. Child, Hospitalized. Dietetic, Prescription. 


\section{REFERÊNCIAS}

1 - Miggiano GA, Carnicelli G. Guidelines for nutritional management in hospitals. Clin Ter 2003; 154: 211-5.

2 - Ferreira HS. Desnutrição, magnitude, significado social e possibilidade de prevenção. Maceió (AL): EDUFAL; 2000.

3 - Monte CMG. Desnutrição: um desafio secular à nutrição infantil. J Pediatr 2000;76 (Supl 3):285-97.

4 - Cortés RV, Nava-Flores G, Pérez CC. Frecuencia de la desnutrición en niños de un hospital pediátrico de tercer nivel. Rev Mexicana Pediatr 1995; 62:131-3.

5 - Batista Filho M, Costa MJ. Desnutrição energetico-proteica experiência do Hospital Universitário de João Pessoa, PB. I - Prevalência de desnutrição. Rev IMIP 1988; 2: 90-3.

6 - Guillerme SN, Taboadela M. Evolucion del estado nutricional de pacientes internados en un hospital pediátrico. Arch Argent Pediatr 1984; 82: 393-402.

7 - Scholfield C, Ashworth A. Por que siguen tan altas lãs tasas de mortalidad por malnutrición grave. Rev Panam Salud Publica 1997; 1: 295-9.

8 - Oliveira AF, Oliveira FLC, Juliano Y, Ancona-Lopez F. Evolução nutricional de crianças hospitalizadas e sob acompanhamento nutricional. Rev Nutr 2005; 18: 341-8.

9 - Sadex MGA, Tudisco ES, Andrade SMB, Manoel NJ, Miszputen SJ, Sigulem DM. Suporte nutricional em pacientes hospitalizados: experiência de um ano. Rev Paul Med 1984; 102: $192-6$.

10 - Waitzberg DL, Caiaffa WT, Correia MITD. Hospital malnutrition: the Brazilian national survey (IBRANUTRI): a study of 4000 patients. Nutrition 2001; 17:573-80.

11 - Kristy MH Christopher D, Linda G. Malnutrition in hospitalized pediatric patients. Arch Pediatr Adolesc Med 1995; 149: 111822.

12 - Institute of Medicine. Dietary Reference Intakes for calcium, phosphorus, magnesium, vitamin D and fluoride. Food and Nutrition Board. Washington, DC: National Academy Press; 1997.

13 - Barr SI, Murphy SP, Poos MI. Interpreting and using the dietary references intakes in dietary assessment of individuals and groups. J Am Diet Assoc 2002; 102:780-8.
14 - Sarni ROS, Souza FIS, Catherino P, Kochi C, Oliveira FLC, Nóbrega FJ. Tratamento da desnutrição em crianças hospitalizadas em São Paulo. Rev Assoc Med Bras 2005; 51: 106-12.

15 - Taylor RM. Preedy VR, Baker AJ, Grimble G. Nutritional support in critically ill children. Clin Nutr 2003; 22: 365-9.

16 - Noether, GE. Introdução à estatística: uma abordagem não paramétrica. Rio de Janeiro, RJ: Guanabara Dois; 1983. p.258.

17 - Alves JGB, Britto LMA, Melo MAS, Oliveira VA. Morbimortalidade do desnutrido grave hospitalizado. J Pediatr 1988; 64: $60-1$.

18 - Manary MJ, Yarasheski KE, Smith S, Abrams ET, Hart CA. Protein quantity, not protein quality, accelerates whole-body leucine kinetics and the acute-phase response during acute infection in marasmic Malawian children. J Nutr 2004; 92:589-95.

19 - Hallberg L, Rossander-Hulten L, Brune M, Gleerup A. Calcium and iron absortion: mechanism of action and nutritional importance. Eur J Clin Nutr 1992; 43:317-27.

20 - Hallberg L, Brune M, Erlandsson M, Sandberg AS, RossanderHulten L. Calcium: effect of different amounts onnonheme and heme - iron absortion in humans. Am J Clin Nutr 1991; 53:112-9.

21 - Thorsdottir I, Gunnarsson BS, Atladottir H, Michaelsen KF, Palsson G. Iron status at 12 months of age - effects of body size, growth and diet in a populationn with hogh birth weight. Eur J Clin Nutr 2003; 57: 505-13.

22 - Grinder-Pederson L, Bukhave K, Jensen M, Hojgaard L, Hansen M. Calcium from milk or calcium-fortified foods does not inhibit nonheme-iron absorption from a whole diet consumed over a 4-d period. Am J Clin Nutr 2004; 80:404-9.

23 - Rogers EJ, Gilbertson HR, Heine RG, Henning R. Barriers to adequate nutrition in critically ill children. Nutrition 2003; 19 865-8.

Recebido em 20/11/2006

Aprovado em 20/04/2007 